\title{
Determination of Intrafraction Laryngeal Movement in Radiotherapy
}

\author{
(Di) İsmail Faruk DURMUŞ, ${ }^{1}$ (D) Bora TAŞ, ${ }^{1}$ ○ Ömer Erol UZEL ${ }^{2}$ \\ 'Department of Radiation Oncology, Yeni Yüzyil Universty Gaziosmanpaşa Hospital, İstanbul-Turkey \\ ${ }^{2}$ Department of Radiation Oncology, İstanbul Cerrahpaşa University Faculty of Medicine, İstanbul-Turkey
}

\begin{abstract}
OBJECTIVE
Movement in early-stage laryngeal radiotherapy is an important factor in the success of the treatment. Thyroid cartilage may move by swallowing, breathing, sound production, and reflexes. During the treatment, the intra-fraction target movement was monitored by СBCT scans. In this study, we investigated the effects of laryngeal movement on the target volume.

\section{METHODS}

CT scans were performed to 16 patients with maximum neck extension and treatment plans were prepared with VMAT fields with 6MV energy. CBCT scanning was performed to all patients before the treatment and necessary corrections were made. Then, simultaneous intrafraction CBCT scanning with the VMAT field was performed during the treatment. When the treatment field was over, the deviation amounts between CT and CBTC in the lateral, vertical and longitudinal axes were determined.
\end{abstract}

\section{RESULTS}

The deviation amount $\leq \pm 0.1 \mathrm{~cm}$ was determined with 293 fractions in the lateral axis, 260 fractions in the vertical axis and 263 fractions in the longitudinal axis. Maximum deviation values were determined as $0.2 \mathrm{~cm}$ in the lateral axis, $0.5 \mathrm{~cm}$ in the vertical axis and $0.5 \mathrm{~cm}$ in the longitudinal axis. If the treatment has a $0.2 \mathrm{~cm}$ CTV-PTV margin (for 305 fractions), treatment can be performed at a confidence interval of $100 \%$ on the lateral axis, $96.1 \%$ on the vertical axis and $94.1 \%$ on the longitudinal axis.

\section{CONCLUSION}

With the help of intra-faction monitoring, we are able to adjust the target margins and doses more precisely in laryngeal radiotherapy, especially for stereotactic treatment. To reduce possible movements in laryngeal radiotherapy, a maximum neck extension should be performed.

Keywords: Intra-fraction motion; intra-fraction CBCT; laryngeal motion.

Copyright $\odot$ 2020, Turkish Society for Radiation Oncology

\section{Introduction}

Early-stage glottic carcinomas are usually confined to the glottic region and are known to be highly localized as there are no lymphatic vessels in the true vocal cords. $[1,2]$ These cancers are normally limited to the mucosa without the involvement of the underlying muscles due to the presence of Reinke's space (superficial lamina propria).[3] Early-stage glottic cancer can be improved by radiotherapy (RT), laser surgery, and surgery; each of these modalities being equally successful concerning local control and cure rates.[4,5] RT is typically delivered 
via parallel-opposed beams to doses between 60 and 70 Gy. Recently, highly conformal techniques, such as intensity-modulated radiotherapy (IMRT) or volumetricmodulated arc therapy (VMAT), have been introduced; however, geometric accuracy has become very critical parallel to the developments in treatment. Modern RT has become a highly complex and precise treatment process with the new imaging modalities, delivery systems, and patient immobilization devices. These technological advances have made it possible to reduce the dose for normal tissue structures and consequently to minimize the risk of toxicity and morbidity, while allowing for dose escalation to the tumour volumes, potentially leading to improved local control.[4-6]

The success of RT treatment is directly related to the delivery of the treatment. Daily set-up variations and internal organ motions may lead to changes in dose distribution and target misalignment. Therefore, all uncertainties with a potential effect on the outcome of the treatment must be determined. The effects of these uncertainties on the planned dose distribution must be as low as possible since the possible mistakes that are to be caused by these uncertainties may change the outcome of the treatment. $[7,8]$ To take these uncertainties into account, appropriate margins around the CTV are used to ensure adequate target coverage. This could result in a significant increase in the irradiated volume and, therefore, a significant additional exposure to healthy tissue. To reduce daily treatment variations, image-guided radiation therapy (IGRT) technology, which provides real-time geometric and anatomic information in the treatment position, has been widely adopted. The reason behind the preference of IGRT is that it increases survival rates and reduces complications by increasing RT precision and accuracy. The concurrent clinical benefit of IGRT for the patient is its ability to monitor the changes in the position and status of the tumour and the patient, which can occur during the course of radiation treatment and to adjust beam delivery accordingly. $[7,8]$ In general, intrafraction adjustments can be grouped into two categories: online and off-line. An online correction takes place when corrections or actions occur at the time of radiation delivery based on predefined thresholds. An off-line approach refers to target tracking without immediate intervention. [7,8] In our study, the movement of the target was considered during irradiation through an off-line approach.

Along with the developing technologies and techniques, the trend towards hypofractionated treatment is increasing. In early stage laryngeal radiotherapy, hypofractionated treatments are an advantageous treat- ment in regards to both shortening of the treatment period and increasing local control. $[9,10]$ In our study, the laryngeal motion was limited to maximum neck extension. Thus, motion management for hypofractionated and stereotactic treatment was determined and also the reliability of safety margins given to PTV during treatment was investigated.

\section{Materials and Methods}

For 16 patients, a three-dimensional planning CT scan was acquired using a multi-slice CT scanner (Biograph $\mathrm{mCT}$, Siemens Medical Solutions) with a $1 \mathrm{~mm}$ slice thickness. In our scan protocol, patients were positioned with the neck extension and a head-neck mask was made. Target volume and organ at risk were contoured. The planning target volume (PTV) was created by adding a $0.2-0.3 \mathrm{~cm}$ isotropic margin around the CTV. According to the Monaco 5.11 treatment planning system, plans were performed using VMAT fields of $200^{\circ}$ or $360^{\circ}$ at $6 \mathrm{MV}$ energies. In the plans, the Monte Carlo algorithm with a dose to medium mode was performed at a grid size of $0.2 \mathrm{~cm}$. Table 1 shows the planning data of 16 patients.

The patients were irradiated in Elekta Versa HD (Elekta, Crawley, UK). This linear accelerator can deliver flattened photon beams (6MV, 10MV, 15MV), flattening filter-free (6MV-FFF, 10MV-FFF) photon beams as well as electron beams. The agility collimator system has a 160 Multi-Leaf Collimator (MLC) system. The MLCs have a width of $0.5 \mathrm{~cm}$, an effective speed of $6.5 \mathrm{~cm} / \mathrm{s}$, and leakage of $0.5 \%$.

In our study, the IGRT system was used as the Elekta X-ray Volume Imaging (XVI) (Elekta, Stockholm, Sweden). A kilo-Voltage Cone Beam computed tomography (kV-CBCT) image set consisting of several twodimensional projection images acquired at different positions around the patient and reconstructed into a $3 \mathrm{D}$ volume. XVI system $\mathrm{kV}-\mathrm{CBCT}$ three-axis image was obtained. The planning CT and CBCT images were compared in XVI and couch shift values in $\mathrm{x}, \mathrm{y}, \mathrm{z}$ and pitch, roll, yaw directions were determined. The necessary couch angle and shift values were set in six dimensions by hexapod couch.[11]

In each fraction, the initial setup was obtained by aligning lasers with pencil markings on the mask. For each patient, two CBCT scans were performed daily. After setting up the patient on the treatment couch with room lasers, the first scan was performed and registered to the reference CT using the thyroid cartilage as the matching structure. In our clinic, according to 
Table 1 Patient characters and plan data

\begin{tabular}{|c|c|c|c|c|c|}
\hline & Dose (Gy) & Fraction & Technic & Stage & Location \\
\hline Patient 1 & $54 / 66 / 67.5$ & 30 & VMAT-SIB & T3N0M0 & Glottic \\
\hline Patient 2 & $54 / 60 / 70$ & 33 & VMAT-SIB & T2NOMO & Glottic \\
\hline Patient 3 & $60 / 66$ & 30 & VMAT-SIB & T1bNOMO & Glottic \\
\hline Patient 4 & $60 / 66$ & 30 & VMAT-SIB & T1NoMo & Glottic \\
\hline Patient 5 & $54 / 60 / 70$ & 33 & VMAT-SIB & T2NOMO & Glottic \\
\hline Patient 6 & $60 / 54$ & 30 & VMAT-SIB & T3N0MO & Glottic \\
\hline Patient 7 & $54 / 60 / 74$ & 33 & VMAT-SIB & T2NOMO & Glottic \\
\hline Patient 8 & $54 / 60 / 70$ & 33 & VMAT-SIB & T2NOMO & Glottic \\
\hline Patient 9 & $54 / 60 / 70$ & 33 & VMAT-SIB & T2N1M0 & Glottic \\
\hline Patient 10 & $54 / 60 / 70$ & 33 & VMAT-SIB & T2NOMO & Glottic \\
\hline Patient 11 & $60 / 67.5$ & 30 & VMAT-SIB & T1bNOMO & Glottic \\
\hline Patient 12 & $52.5 / 60 / 66$ & 30 & VMAT-SIB & T2NOMO & Glottic \\
\hline Patient 13 & 63 & 28 & VMAT & T1bNOMO & Glottic \\
\hline Patient 14 & 57.6 & 16 & VMAT & TisNOMO & Glottic \\
\hline Patient 15 & 57.6 & 16 & VMAT & T1bNOMO & Glottic \\
\hline Patient 16 & 57.6 & 16 & VMAT & T1aNOMO & Glottic \\
\hline
\end{tabular}

VMAT: Volumetric-modulated arc therapy; SIB: Simultaneous integrated boost

XVI "head and neck" Protocol (100 kV, $18.3 \mathrm{mAs}), 2 \mathrm{D}$ portal projections were taken in a $200^{\circ}$ gantry angle range, and $3 \mathrm{D}$ volumes were constructed. In the second scan, a 2D projection was scanned on the XVI system synchronously with the VMAT field during the treatment and 3D images were obtained. According to the XVI “intrafraction" Protocol (120 kV, $146.4 \mathrm{mAs}), 2 \mathrm{D}$ portal projections were scanned at $200^{\circ}$ or $360^{\circ}$ angle and $3 \mathrm{D}$ images were obtained. In the second scan, the intrafraction CBCT was matched with the reference CT and the amount of thyroid cartilage movement during the treatment was determined. Deviations in the leftright (LR), craniocaudal (CC) and anterior-posterior (AP) directions were obtained. Thus, any motion that could occur in the target volume during irradiation was detected (Fig. 1).

\section{Results}

This study investigated intrafractional movements through CBCT scanning in 305 fractions for 16 patients during treatments. Real-time tumour guidance was performed by concurrent intrafraction CBCT scan at the beginning of the treatments. When the treatment was completed, the cartilage position was checked again, and positional deviations were determined in three directions. These deviations are shown in Table 2.

Generally, a deviation of less than $0.1 \mathrm{~cm}$ was observed in the LR, CC and AP directions during the
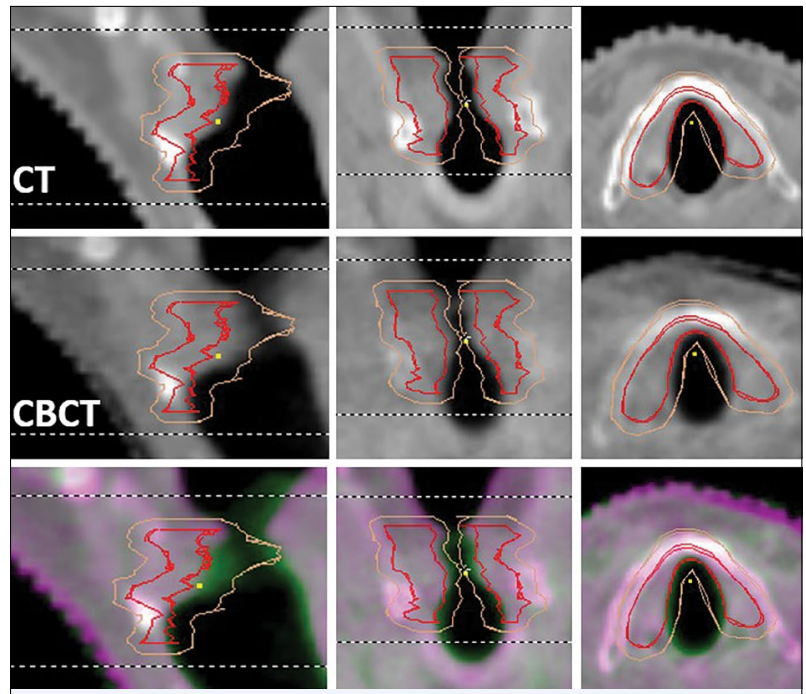

Fig. 1. $\mathrm{CT}$, intrafraction $\mathrm{CBCT}$, and matched images.

treatment. The vertical and longitudinal directions showed a higher deviation than the lateral direction. Absolute averages (plus and minus direction independent) were determined as $0.022 \pm 0.04 \mathrm{~cm}$ in the LR direction, $0.064 \pm 0.09 \mathrm{~cm}$ in the CC direction and $0.077 \pm 0.08 \mathrm{~cm}$ in the AP direction. Absolute maximum deviation values (plus and minus direction independent) were $0.2 \mathrm{~cm}$ in the LR direction, $0.5 \mathrm{~cm}$ in the $\mathrm{CC}$ direction, and $0.5 \mathrm{~cm}$ in the AP direction. Figures 2,3 , and 4 show the directions and quantities of deviation in 305 fractions. 
Table 2 Fraction numbers according to deviation amounts in three axes

\begin{tabular}{lccc} 
& Left-right & Cranio-caudal & Antero-posterior \\
\hline$a>+0.3 \mathrm{~cm}$ & - & 4 & 1 \\
$+0.2 \mathrm{~cm}<\mathrm{a} \leq+0.3 \mathrm{~cm}$ & - & 8 & 8 \\
$+0.1 \mathrm{~cm}<\mathrm{a} \leq+0.2 \mathrm{~cm}$ & 6 & 20 & 23 \\
$+0,1 \mathrm{~cm} \geq a \geq-0.1 \mathrm{~cm}$ & 293 & 263 & 260 \\
$-0.2 \mathrm{~cm} \geq a>-0.1 \mathrm{~cm}$ & 6 & 4 & 10 \\
$-0.3 \mathrm{~cm} \geq a>-0.2 \mathrm{~cm}$ & - & 5 & 2 \\
$a<-0.3 \mathrm{~cm}$ & - & 1 & 1 \\
\hline
\end{tabular}

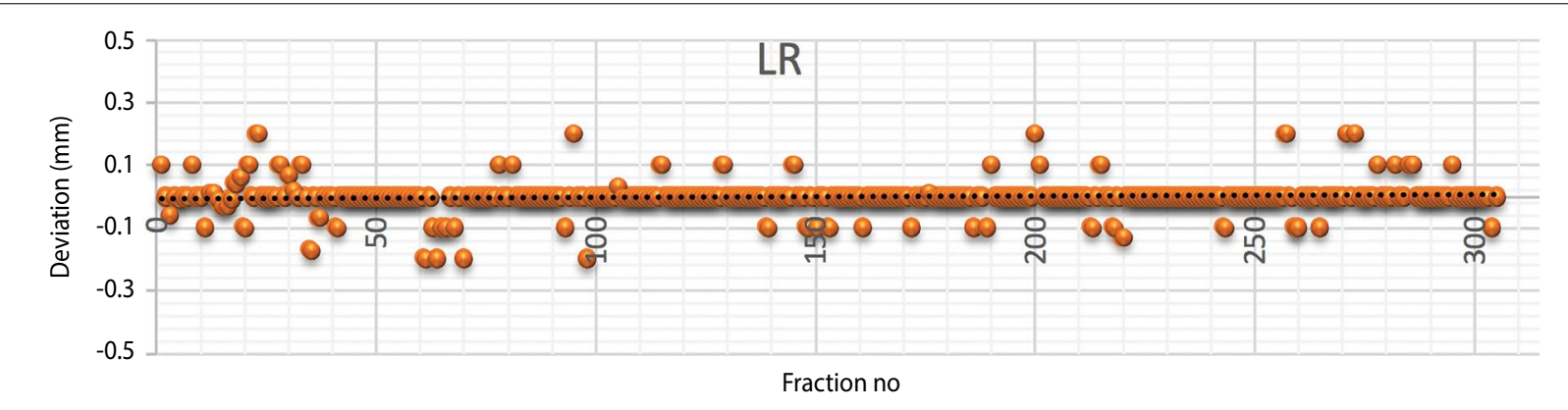

Fig. 2. Lateral deviations in 305 fractions.

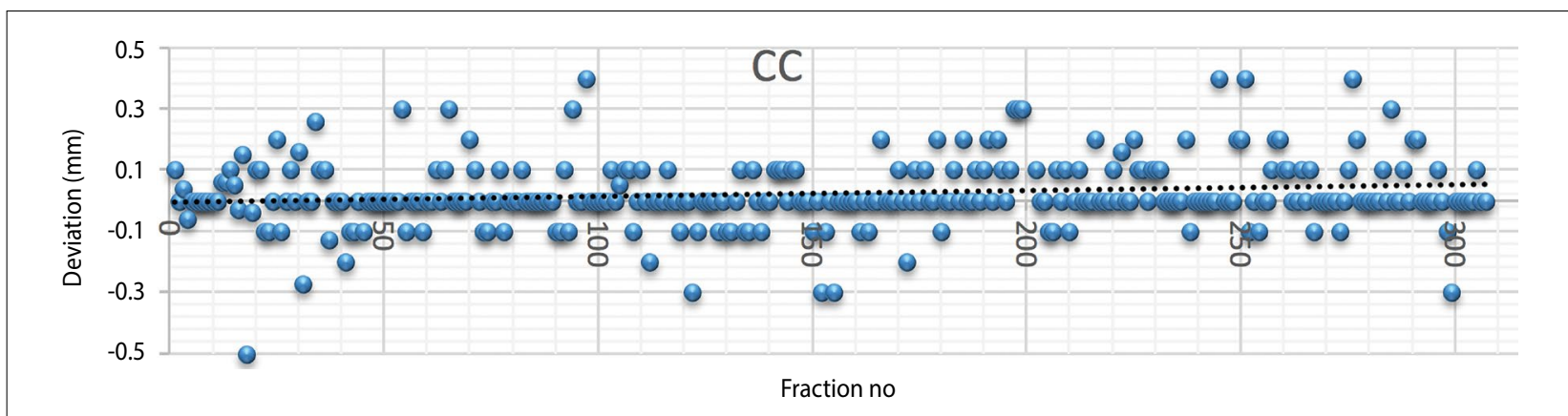

Fig. 3. Longitudinal deviations in 305 fractions.

\section{Discussion}

Intrafraction guide in laryngeal radiotherapy allows us to adjust target margins and target dose more precisely, especially for stereotactic treatments and controls the maximum deviation of the target for each fraction. Although the image quality of pre-treatment-CBCT and intrafraction-CBCT scans were lower than the image quality of planning CT, generally, a deviation of less than $1 \mathrm{~mm}$ was observed in the LR, CC and AP directions during treatment. In Kwa et al.s[12] study, single vocal cord of T1a for inter and intra-tracheal target motions in laryngeal tumours for 42 patients was examined. It was found that $3 \mathrm{~mm}, 5 \mathrm{~mm}$ and $3 \mathrm{~mm}$ PTV margins were added in sufficient image guidance and LR, CC and AP directions to ensure excellent target coverage in a single vocal cord. In Kauweloa et al.s[13] study, the respiratory-induced larynx movement in the HYDRA (Phase 1 Trial of Stereotactic Hypo-fractionated Radio-ablative Treatment of Laryngeal Cancer) studies was examined. This study aimed to evaluate laryngeal movements by four-dimensional computed tomography and breathhold technique and to determine PTV margins. The laryngeal motion on daily CBCT images was obtained as a maximum of $7.21 \mathrm{~mm}$. Although daily treatment verification for breath-hold patients was up to $7.21 \mathrm{~mm}$ interfaction laryngeal movement, intrafractional larynx mo- 


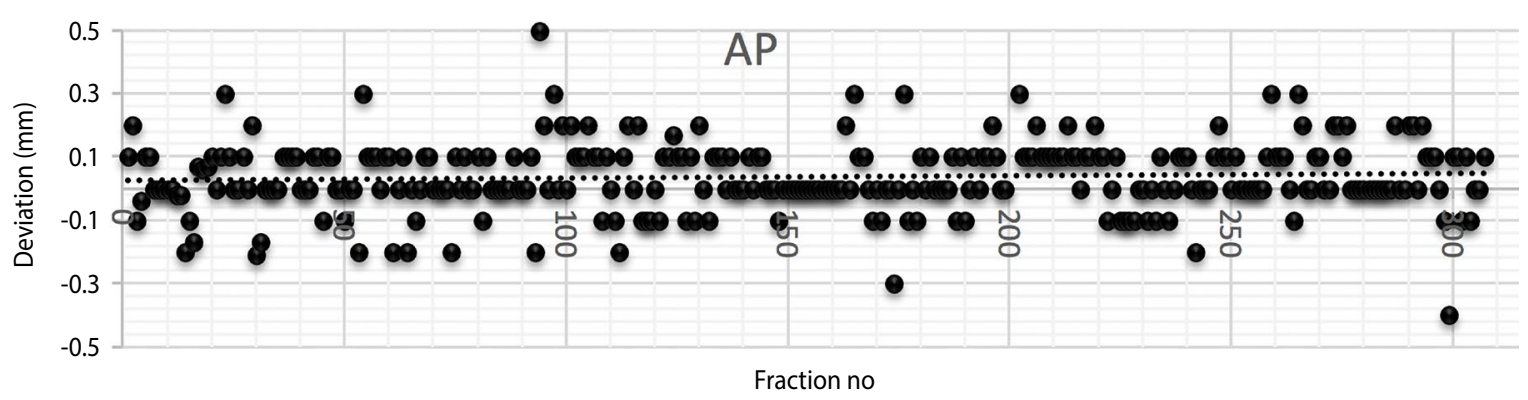

Fig. 4. Vertical deviations in 305 fractions.

tion was less than $2.1 \mathrm{~mm}$. In our study, we observed less intrafraction movement with maximum neck extension. Baron et al.s.'14] study aimed to determine the setup errors and the required CTV-PTV margins depending on the larynx movement. In this study, images of $7 \mathrm{HNC}$ patients who received radiation therapy with daily CT monitoring were used. Daily patient CT on rails scans were imported into a treatment planning system, and both $\mathrm{C} 5$ and the thyroid cartilage were auto-segmented. The average mean displacement for each anatomical point was $4.1 \mathrm{~mm}$ (range, 2.7-5.1), the average systematic error for each point was $1.2 \mathrm{~mm}$ (range, 0.86-1.5), the average random error for each point was $2.1 \mathrm{~mm}$ (range, 1.3-2.8), and the average CTV-PTV margin for each point was $4.4 \mathrm{~mm}$ (range, 3.3-5.7). Larynx motion is a significant cause of RT setup/delivery error in HNC when the larynx is either in a CTV or OAR. The need for uniform expansion of at least $6 \mathrm{~mm}$ to compensate for larynx motion was estimated. In Gangsaas et al.s[15] study, the variation of inter-faction setup for primary tumours, elective nodes and vertebrae in laryngeal cancer patients were investigated and the validity of CBCT guided correction protocols was examined. Because of the poor correlation between displacements of the primary CTV and the vertebrae, even with daily online repositioning of the vertebrae, the required margin around the primary CTV was $6.9 \mathrm{~mm}$. Laryngeal cancer patients showed substantial inter-fraction setup variations, including large time trends and poor correlation between primary tumour displacements, and the motion of the nodes and vertebrae. In Bahig et al's[16] study, the movement of the larynx in twenty patients was examined. Mean amplitude of larynx swallowing excursion was obtained $23 \mathrm{~mm}$ and $6 \mathrm{~mm}$ in the superior and anterior directions. The mean breathing movement was found to reach $4 \mathrm{~mm}$ and $2 \mathrm{~mm}$ in the superior-inferior and antero-posterior directions. In our study, swallowing is prevented by maximum neck extension. In
Bruijnen at al.s[17] study, eighty-four head and neck patients with 3T MRI were examined. Approximately five minutes after the scan, the tumour was scanned for one minute with the $2 \mathrm{D}$ sagittal cine-mode. Therefore, the intra-fraction movements were detected. The movement of the intra-fraction tumour to its origin was calculated and PTV margins were re-calculated. As a result of the study, the margin recipe primarily expanded the PTV approximately $0.2 \mathrm{~cm}$ for laryngeal tumours. Movement in the vocal cord is caused by swallowing, sound production, breathing and reflexes. In our clinic, even though the mask is made according to the maximum neck extension, there may still be movement. In general, less than $0.1 \mathrm{~cm}$ of motion on average is observed in all three dimensions in our study. In 305 fractions for 16 patients, if the CTV-PTV safety margin was $1 \mathrm{~mm}$, it could be treated with confidence interval of $96 \%$ in the lateral direction, $86.2 \%$ in the longitudinal direction and $85.2 \%$ in the vertical direction. If the CTV-PTV safety margin was $0.2 \mathrm{~cm}$, it could be treated in the lateral direction at $100 \%$, in the longitudinal direction $94.1 \%$ and in the vertical direction $96.1 \%$, with a confidence interval. More deviations were observed in vertical and longitudinal directions due to cartilage movement.

\section{Conclusion}

For each patient, the maximum movement of the target volume should be determined in the first days of treatment. And if necessary, the safety margins must be readjusted. In our study, the maximum neck extension is restricted with motion and even if there is a maximum deviation of $2 \mathrm{~mm}$ with intrafractional monitoring, we treat with a confidence interval of over $94 \%$. Thus, it is a guiding study for hypofractionated/ stereotactic treatments. Swallowing is rare and fast. If swallowing occurs during the performance of a CT scan, there is a systematic risk of targeting errors. To 
minimize the possibility of this error and the possibility of other sources of motion as well, the mask should be made with maximum neck extension. As a result of our study in our clinic, movement is restricted with maximum neck extension, and CTV-PTV is $0.2 \mathrm{~cm}$ margin. The possible systematic and random errors are prevented by the intrafraction CBCT guide.

In stereotactic treatments, the motion should be restricted in to reduce possible errors. The possible amount of movement must be determined and the safety margins must be adjusted accordingly.

Peer-review: Externally peer-reviewed.

Conflict of Interest: None declared.

Financial Support: None declared.

Authorship contributions: Concept - İ.F.D., Ö.E.U.; Design - İ.F.D., B.T., Ö.E.U.; Supervision - İ.F.D.; Funding - None; Materials - İ.F.D., B.T., Ö.E.U.; Data collection and/or processing - İ.F.D., B.T., Ö.E.U.; Data analysis and/or interpretation İ.F.D., B.T., Ö.E.U.; Literature search - İ.F.D.; Writing - İ.F.D., B.T., Ö.E.U.; Critical review - İ.F.D., B.T., Ö.E.U.

\section{References}

1. Mittal B, Rao DV, Marks JE, Perez CA. Role of radiation in the management of early vocal cord carcinoma. Int J Radiat Oncol Biol Phys 1983;9(7):997-1002.

2. Werner JA, Schünke M, Rudert H, Tillmann B. Description and clinical importance of the lymphatics of the vocal fold. Otolaryngol Head Neck Surg 1990;102(1):13-9.

3. Pradhan S, Kannan R. Surgery for laryngeal cancer: anatomy and patterns of spread. Voice conservation surgery for laryngeal and hypopharyngeal cancer. Mumbai: Lloyds Publishing House; 2006. p. 10-5.

4. Licitra L, Bernier J, Grandi C, Locati L, Merlano M, Gatta G, et al. Cancer of the larynx. Crit Rev Oncol Hematol 2003;47(1):65-80.

5. Levendag PC, Teguh DN, Keskin-Cambay F, AlMamgani A, van Rooij P, Astreinidou E, et al. Single vocal cord irradiation: A competitive treatment strategy in early glottic cancer. Radiother Oncol 2011;101(3):415-9.

6. Matthew CW, Yvonne DP, Rupesh K, Sara JZ, Eric M, John FG. Clinical and dosimetric implications of intensity-modulated radiotherapy for early-stage glottic carcinoma. Medical Dos 2015;41:64-9.

7. AAPM. The role of in-room $\mathrm{kV} X$-ray imaging for patient setup and target localization. AAPM TG-104. Madison (WI): Medical Physics Publishing; 2009.
Available at: http://www.aapm.org/pubs/reports/. Accessed Feb 4, 2020.

8. Seuntjens J, Lartigau EF, Cora S, Ding GX, Nuyttens J, Roberge $\mathrm{D}$, et al. Prescribing, recording, and reporting of stereotactic treatments with small photon beams. ICRU report 91. J ICRU 2014;14(2):1-160.

9. Gowda RV, Henk JM, Mais KL, Sykes AJ, Swindell R, Slevin NJ. Three Weeks Radiotherapy for T1 Glottic Cancer: Christie and Royal Marsden Hospital Experience. Radiother Oncol 2003;68(2):105-11.

10. Ermiş E, Teo M, Dyker KE, Fosker C, Sen M, Prestwich RJ. Definitive hypofractionated radiotherapy for early glottis carcinoma: experience of $55 \mathrm{~Gy}$ in 20 fractions. Radiat Oncol 2015;10:203.

11. Elekta XVI R 5.0; Instructions for Use. Published July 2013. Available at: http://ekstern.infonet.regionsyddanmark.dk/files/Formularer/Upload/2016/02/xvi\%20 R5.0_1501452\%2002.pdf. Accessed Feb 4, 2020.

12. Kwa SL, Al-Mamgani A, Osman SO, Gangsaas A, Levendag PC, Heijmen BJ. Inter- and intrafraction target motion in highly focused single vocal cord irradiation of tla larynx cancer patients. Int J Radiat Oncol Biol Phys 2015;93(1):190-5.

13. Kauweloa K, Wang H, Garden AS, Chi PCM, Rosenthal DI, Fuller CD, et al. Evaluation of the Larynx Motion Using Four- Dimensional and Breath-Hold Computed Tomography (Poster No: 3615). International Journal of Radiation Oncology, Biology, Physics 2017;99(Supple 2):E677.

14. Baron CA, Awan MJ, Mohamed AS, Akel I, Rosenthal DI, Gunn GB, et al. Estimation of daily interfractional larynx residual setup error after isocentric alignment for head and neck radiotherapy: quality assurance implications for target volume and organs-at-risk margination using daily CT on- rails imaging. J Appl Clin Med Phys 2014;16(1):5108.

15. Gangsaas A, Astreinidou E, Quint S, Levendag PC, Heijmen B. Cone-beam computed tomographyguided positioning of laryngeal cancer patients with large interfraction time trends in setup and nonrigid anatomy variations. Int J Radiat Oncol Biol Phys 2013;87(2):401-6.

16. Bahig H, Nguyen-Tan PF, Filion É, Roberge D, Thanomsack P, de Guise J, et al. Larynx motion considerations in partial larynx volumetric modulated arc therapy for early glottic cancer. J Med Imaging Radiat Oncol 2017;61(5):666-73.

17. Bruijnen T, Stemkens B, Terhaard C, Lagendijk JJW, Raaijmakers CP, Tijssen RH. MRI-Based Radiation Therapy: Intrafraction Motion Quantification of Head and Neck Tumors Using Cine Magnetic Resonance Imaging. International Journal of Radiation Oncology, Biology, Physics. 2018;100(5):1358. 